\title{
Background Rates of Acute Mountain Sickness-Like Symptoms at Low Altitude in Adolescents Using Lake Louise Score
}

Jon Dallimore, MB, BS, MSc, MRCGP, MCEM, DCH, DRCOG, FFTM RCPS (Glasg); Jo-ai Foley, MB, ChB; Peter Valentine, BM, BS, B Med Sci

From the Department of Emergency Medicine, Bristol Royal Infirmary, Bristol, UK (Dr Dallimore); SHO, Emergency Medicine, Musgrove Park Hospital, Taunton, UK (Dr Foley); and CT1, Anaesthetics, Horton Hospital, Banbury, UK (Dr Valentine).

Objective.-To record the incidence of symptoms consistent with a diagnosis of acute mountain sickness (AMS), using the Lake Louise questionnaire, in adolescents hiking at low altitude.

Methods.-The study was carried out on a cohort of 123 adolescents during a 3-day trip on Dartmoor, UK, at an altitude of less than $500 \mathrm{~m}$. The incidence of symptoms experienced was measured using the Lake Louise questionnaire, which was completed twice daily by each participant. An episode consistent with AMS, but in the low altitude setting, was defined as a score of 3 or more on the Lake Louise questionnaire in the presence of a headache.

Results.-There were 59 boys and 64 girls in the study with an average age of 16.7 years. The response rate was $100 \%$. A total of 59 episodes of scores consistent with AMS was recorded during the 3-day period. Forty-two of these episodes were reported by girls (71\%). AMS scores between 3 and 8 were recorded, and the daily incidence rates of scores consistent with AMS but at low altitude were between $7.3 \%$ and $11.3 \%$.

Conclusions.-This study shows that adolescents at low altitude reported a background incidence of symptoms that at high altitude would lead to a diagnosis of AMS.

Key words: acute mountain sickness, altitude, adolescents, Lake Louise score

\section{Introduction}

People traveling to high altitude often experience symptoms known as acute mountain sickness (AMS). AMS is a well-recognized phenomenon, which has been extensively documented in a range of age groups at various altitudes worldwide. The relative incidence of AMS in adolescents, compared with adults, still remains controversial. ${ }^{1}$ Many studies have used the Lake Louise questionnaire score ${ }^{2}$ to confirm a diagnosis of AMS.

In this study we propose that adolescents might experience symptoms during outdoor activities at low altitude similar to those of AMS. Using the Lake Louise Score out of context in the low altitude setting, it might be possible to attain a score consistent with AMS. If this occurs it would imply that these background symptoms

Corresponding author: Dr Jo-ai Foley, MB, ChB, Medical Assessment Unit, Derriford Hospital, Crownhill, Plymouth, Devon PL6 8DH, United Kingdom (e-mail: joaifoley@googlemail.com). should be accounted for when attempting to diagnose AMS at altitude using the Lake Louise Score.

Acute mountain sickness is commonly experienced by people traveling to high altitudes, typically above $2400 \mathrm{~m}^{3}$ The syndrome is characterized by symptoms of headache, nausea or vomiting, dyspnea, fatigue, poor appetite, dizziness, and difficulty in sleeping. ${ }^{4}$ In 1991, in an attempt to standardize the assessment of AMS, the Lake Louise questionnaire scoring system was developed by the International Hypoxia Symposium. ${ }^{2}$ This defined the diagnosis of AMS as the presence of 3 or more of the above symptoms, including a headache, in the context of a recent gain in altitude.

There have been many studies recording the incidence of AMS and research into methods of predicting AMS in adult travelers. However, from the limited research to date, the incidence of AMS in adolescents is generally regarded as being higher and more variable in range compared with adults. A study by Moraga et $\mathrm{al}^{5}$ in 
Northern Chile at $4400 \mathrm{~m}$ found the incidence of AMS in adolescents (13-18 years) to be higher, at a rate of $50 \%$, when compared with the incidence of $27 \%$ observed in adults. A larger study by Honigman et $\mathrm{al}^{6}$ observed similar findings in the Rocky Mountains of Colorado (elevations of $1920 \mathrm{~m}-2957 \mathrm{~m}$ ) with an incidence of $45 \%$ in those aged 16 to 19 years old (compared with an overall average of $25 \%$ ). A more recent study found that 91.7\% of adolescents had Lake Louise Scores consistent with AMS when traveling to $5500 \mathrm{~m}$ in Peru. ${ }^{7}$

Owing to such varying rates, the relative incidence of AMS in adolescents compared with adults remains controversial. The higher incidence has been attributed to several factors causing AMS-like symptoms and thereby falsely elevating Lake Louise Scores. Some studies ${ }^{8,9}$ propose important unrelated factors such as anxiety, travel, intercurrent illness, diet, and disruption of daily routine might be responsible.

The pathophysiological changes associated with AMS in adolescents may be a contributing factor because physiology alters with age. In two reports by Moraga et al, ${ }^{5,9}$ the data showed children to be more sensitive to hypobaric hypoxia than their parents after rapid ascent to $3500 \mathrm{~m}$ in Chile and in a similar study in northern Chile to elevations of $4400 \mathrm{~m}$. The results demonstrated that children, and adolescents to a lesser extent, are more sensitive to hypoxia during altitude ascent, manifesting as symptoms of AMS (measured by the Children's Lake Louise Score as devised by Yaron $^{10}$ ) and significant desaturation. Therefore, both of these papers ${ }^{5,9}$ support the theory that an exaggerated hypoxemic response to high altitude exposure in younger adolescents gives them a higher susceptibility to AMS than adults.

The aim of this study was to examine the incidence of symptoms experienced by a group of adolescents (13-19 years) during a 3-day hike at low altitude on Dartmoor, UK. The research aim was to investigate the background incidence of AMS-like symptoms in adolescents traveling at low altitude. A high incidence of generalized symptoms pertaining to those of the Lake Louise Score at low altitude would then question the specificity of the Lake Louise Score when used to diagnose AMS in adolescents traveling at altitude. It might also explain the relatively high rates of AMS diagnosed in other studies examining this age group.

\section{Methods}

\section{STUDY GROUP}

This was a prospective, descriptive study of 123 adolescents (64 girls and 59 boys) aged between 14 and 19 years (average, 16.7 years) during a training course based on Dartmoor, UK, in March 1998. The participants were split into 11 groups. Each group was given a verbal briefing about the study and told that the study was designed to examine the symptoms of altitude sickness that may also occur at low elevations during a short trekking trip. All participants were given an information sheet and signed a consent form before taking part. At the time the study was conducted the work was exempt from the requirement for institutional review board approval. For 3 days and 2 nights the participants camped and hiked at altitudes of less than $500 \mathrm{~m}$, while carrying all food, tents, and equipment. At all times the subjects were supervised by adult leaders and were not permitted to take alcohol or to use narcotics.

\section{ACUTE MOUNTAIN SICKNESS QUESTIONNAIRE}

Each individual was given a Lake Louise self-reporting questionnaire to be completed twice daily, although it was noted as being used out of context as there was no recent gain in altitude for the participants during the study. Details of age and gender were recorded on the sheets. A single observer (JD) visited the campsites each morning and evening to issue the Lake Louise questionnaires. The Lake Louise Scores were calculated in the morning and evening for each individual. An episode consistent with AMS was defined as a score of 3 or more on the Lake Louise questionnaires in the presence of a headache. Data were entered on Microsoft Excel (Microsoft Corp, Redmond, WA) spreadsheets to aid analysis.

\section{Results}

The response rate was $100 \%$ on each of the planned 5 recording sessions. There were 59 boys and 64 girls in the study, with an average age of 16.7 years. A total of 59 episodes of scores consistent with AMS was recorded during the 3-day period. Forty-two of these episodes were reported by girls ( $71 \%$ of the total).

In this study $7.3 \%$ of participants had Lake Louise Scores compatible with AMS on arrival on Dartmoor. This increased to $8.9 \%$ after a short walk, decreased after a night's rest to $8.1 \%$, and then peaked to an incidence of $11.3 \%$ on the evening of the second day. After the second night's camp, $8.1 \%$ of subjects recorded symptoms compatible with AMS. Tiredness and poor sleep were the predominant symptoms, in addition to headache contributing to these scores. Tiredness was reported in $88 \%$ and poor sleep in $69 \%$.

The mean AMS scores varied from 3.8 to 4.4 (range, 3-8). Although the group recorded incidences of AMS between $7.3 \%$ and $11.3 \%$ (see Figure), it should be noted that the subjects did not meet all the criteria for the 


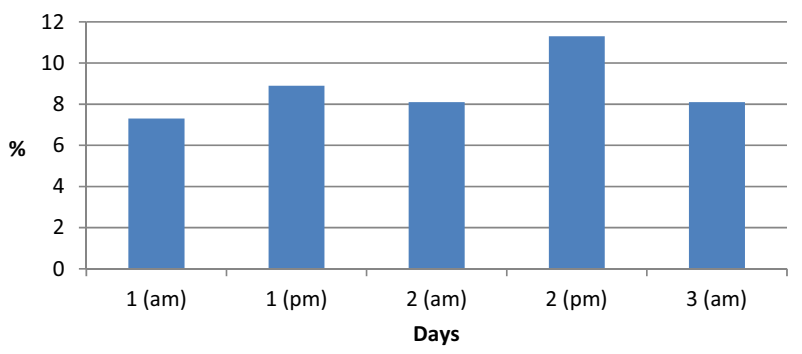

Figure. Percentage incidence of scores suggesting acute mountain sickness (AMS).

definition of AMS as the symptoms did not occur "in the setting of a recent gain in altitude."

\section{Discussion}

The daily incidence of scores consistent with a diagnosis of AMS, but at low altitude, was between $7.3 \%$ and $11.3 \%$ for this adolescent cohort. These results show that a significant rate of background symptoms is experienced by adolescents at low altitude. At high altitude these scores would have led to a diagnosis of AMS. This high rate of potentially false-positive results may explain, in part, why there are such varied results found in the reported incidence of AMS in adolescents.

This study group recorded tiredness and poor sleep as their predominant symptoms. These symptoms, as well as headaches, loss of appetite, and dizziness, all have a background rate in any population and may be provoked by factors such as travel, change in routine, and unaccustomed physical exercise. It is therefore not surprising that there is a background rate of AMS-like symptoms, even at low altitude.

To date there are no directly comparable data examining the incidence and severity of AMS symptoms in this age group. Similar high background rates of AMSlike symptoms have been found in other studies, most notably by Theis et $\mathrm{al}^{8}$ who examined two groups of similarly aged children, one at sea level (405 individuals) and one ascending at altitude from 1600 to $2835 \mathrm{~m}$ (558 individuals). The data showed that $28 \%$ of the group at altitude experienced symptoms compatible with AMS. It was suggested that a large proportion of these symptoms present were attributable to factors other than altitude, such as travel, anxiety, or disruption of daily routine, as the group at sea level also recorded a high incidence of scores compatible with AMS in $21 \%$ of cases. ${ }^{8}$

The Lake Louise scoring system identifies symptoms that could describe a number of different illnesses or simply fatigue after physical exertion. An International Consensus Statement by an Ad Hoc Committee of the International Society for Mountain Medicine 2001 on
"Children at High Altitude" acknowledged that often the signs and symptoms of AMS are nonspecific and can easily be confused with unrelated variables such as intercurrent illness, diet, and psychological factors. ${ }^{11}$ The Lake Louise questionnaire's "defining symptoms" of AMS are therefore not specific, so it is important that other factors and differential diagnoses be considered, which may account for the symptoms experienced.

Other methods have been used to diagnose AMS. In 1976 Hackett et al ${ }^{12}$ described an AMS evaluation system that consisted of a short, structured interview and physical examination. This method was suggested by Roeggla et $\mathrm{al}^{13}$ to be the gold standard for evaluating the incidence of AMS. Another scoring system called the Environmental Statistical Questionnaire III (ESQ III), which consists of a 67-question symptom inventory, was used in a study by Kayser. ${ }^{14}$ Although the Lake Louise questionnaire is considered to overestimate the incidence of AMS, when compared with the Hackett or ESQ III diagnostic tools, the complexity of these other scoring systems undermines their practicality and feasibility as methods of diagnosing AMS.

Differential diagnoses such as viral upper respiratory infections are common in trekkers at high altitude and present with symptoms very similar to those which suggest AMS. A study by Murdoch ${ }^{15}$ analyzed symptoms of infection and altitude illness among hikers in the Mount Everest region of Nepal. He found that $87 \%$ experienced at least one symptom of "infection," and of the study group, 57\% also had AMS using the Lake Louise diagnostic criteria.

The limitations to this study include its relatively small sample size, although it is the largest study of adolescents at low altitude to date. A larger study would help to determine whether a difference between genders exists. The subjects were not blinded and were informed that the aim of the study was to examine the incidence of general symptoms that may be experienced at high altitude. The report did not account for any predisposing factors, eg, smoking status; comorbidities, eg, asthma; or other variables such as baseline fitness that the individual participants may have had. All of these may have influenced the incidence rates calculated.

\section{Conclusions}

This study has demonstrated that adolescents can experience a background incidence of AMS-like symptoms while traveling at low altitude, which give Lake Louise Scores consistent with AMS. The Lake Louise questionnaire is a relatively sensitive tool; however, specificity is variable, and false-positive results are likely to occur. As a result, the data confirm that the Lake Louise question- 
naire has a tendency to overestimate the frequency of diagnosing AMS in adolescents.

Although there is no highly specific and sensitive tool to assess and predict AMS in adolescents, researchers still conclude that the Lake Louise questionnaire is a valuable tool to allow simple and rapid self-assessment of AMS, ${ }^{14,16}$ even in adolescents. It is very important to diagnose AMS, and it is better that an assessment tool is more sensitive, even if it is less specific. Those trekking to high altitudes should be aware that background rates of AMS-like symptoms need to be taken into account when using the Lake Louise questionnaire before diagnosing true AMS. However, trekkers should always remain vigilant and have a low threshold for diagnosing and treating AMS. It is more sensible to assume a diagnosis of AMS rather than to attribute the symptoms to something less potentially dangerous.

\section{References}

1. Dallimore J, Rowbotham EC. Incidence of acute mountain sickness in adolescents. Wilderness Environ Med. 2009;20: 221-224.

2. Sutton JR, Coates G, Houston CS. Lake Louise consensus on definition and quantification of altitude illness. In: Sutton JR, Coates G, Houston CS, eds. Hypoxia and Mountain Medicine. New York, NY: Pergamon Press; 1991: 327-330.

3. Roach R, Stepanek J, Hackett, P. Acute Mountain Sickness and High-Altitude Cerebral Edema. In: Pandolf KB, Burr RE, eds. Medical Aspects of Harsh Environments. Vol. 2. Washington, DC: Department of the Army, Office of The Surgeon General, Borden Institute; 2002:765-794.

4. Barry PW, Pollard AJ. Altitude illness. BMJ. 2003;326: 915-919.

5. Moraga, FA, Osorio JD, Vargas ME. Acute mountain sickness in tourists with children at Lake Chungará (4400 m) in northern Chile. Wilderness Environ Med. 2002;13: 31-35.
6. Honigman B, Theis MK, Koziol-McLain J, et al. Acute mountain sickness in a general tourist population at moderate altitudes. Ann Intern Med. 1993;118:587-592.

7. Imray CHE, Kennedy $\mathrm{CH}$, Pattinson $\mathrm{K}$, Brearey $\mathrm{S}$, Wright A; Birmingham Medical Research Expeditionary Society. Self-assessment of acute mountain sickness in adolescents: a pilot study. Wilderness Environ Med. 2004; 15:202-206.

8. Theis MK, Honigman B, Yip R, McBride D, Houston CS, Moore LG. Acute mountain sickness in children at 2835 meters. Am J Dis Child. 1993;147:143-145.

9. Moraga FA, Pedreros CP, Rodríguez. Acute mountain sickness in children and their parents after rapid ascent to 3500 m (Putre, Chile). Wilderness Environ Med. 2008;19: 287-292.

10. Yaron M, Waldman N, Niermeyer S, Nicholas R, Honigman B. The diagnosis of acute mountain sickness in preverbal children. Arch Pediatr Adolesc Med. 1998;152: 683-687.

11. Pollard AJ, Niermeyer S, Barry P, et al. Children at high altitude: an international consensus statement by an ad hoc committee of the International Society for Mountain Medicine, March 12, 2001. High Alt Med Biol. 2001;2: 389-403.

12. Hackett PH, Rennie D, Levine HD. The incidence, importance, and prophylaxis of acute mountain sickness. Lancet. 1976;2:1149-1155.

13. Roeggla G, Roeggla M, Podolsky A, Wagner A, Laggner AN. How can acute mountain sickness be quantified at moderate altitude? J R Soc Med. 1996;89:141-143.

14. Kayser B. Acute mountain sickness in western tourists around the Thorong Pass $(5,400 \mathrm{~m})$ in Nepal. $J$ Wilderness Med. 1991;2;110-117.

15. Murdoch DR. Symptoms of infection and altitude illness among hikers in the Mount Everest region of Nepal. Aviat Space Environ Med. 1995;66:148-151.

16. Savourey G, Guinet A, Besnard Y, Garcia N, Hanniquet AM, Bittel J. Evaluation of the Lake Louise acute mountain sickness scoring system in a hypobaric chamber. Aviat Space Environ Med. 1995;66:963-967. 